\title{
Topotaxis of active Brownian particles
}

\author{
Koen Schakenraad, ${ }^{1,2}$ Linda Ravazzano, ${ }^{1,3}$ Niladri Sarkar $\odot,{ }^{1}$ Joeri A. J. Wondergem $\odot,{ }^{4}$ \\ Roeland M. H. Merks $\odot,{ }^{2,5}$ and Luca Giomi $\odot^{1, *}$ \\ ${ }^{1}$ Instituut-Lorentz, Leiden University, P.O. Box 9506, 2300 RA Leiden, The Netherlands \\ ${ }^{2}$ Mathematical Institute, Leiden University, P.O. Box 9512, 2300 RA Leiden, The Netherlands \\ ${ }^{3}$ Center for Complexity and Biosystems, Department of Physics, University of Milan, Via Celoria 16, 20133 Milano, Italy \\ ${ }^{4}$ Kamerlingh Onnes-Huygens Laboratory, Leiden University, P.O. Box 9504, 2300 RA Leiden, The Netherlands \\ ${ }^{5}$ Institute of Biology, Leiden University, P.O. Box 9505, 2300 RA Leiden, The Netherlands
}

(Received 16 August 2019; accepted 26 January 2020; published 3 March 2020)

\begin{abstract}
Recent experimental studies have demonstrated that cellular motion can be directed by topographical gradients, such as those resulting from spatial variations in the features of a micropatterned substrate. This phenomenon, known as topotaxis, has been extensively studied for topographical gradients at the subcellular scale, but can also occur in the presence of a spatially varying density of cell-sized features. Such a large-scale topotaxis has recently been observed in highly motile cells that persistently crawl within an array of obstacles with smoothly varying lattice spacing. We introduce a toy model of large-scale topotaxis, based on active Brownian particles. Using numerical simulations and analytical arguments, we demonstrate that topographical gradients introduce a spatial modulation of the particles' persistence, leading to directed motion toward regions of higher persistence. Our results demonstrate that persistent motion alone is sufficient to drive large-scale topotaxis and could serve as a starting point for more detailed studies on self-propelled particles and cells.
\end{abstract}

DOI: 10.1103/PhysRevE.101.032602

\section{INTRODUCTION}

Whether in vitro or in vivo, cellular motion is often biased by directional cues from the cell's microenvironment. Chemotaxis, i.e., the ability of cells to move in response to chemical gradients, is the best known example of this functionality and plays a crucial role in many aspects of biological organization in both prokaryotes and eukaryotes [1,2]. Yet, it has become increasingly evident that in addition to chemical cues, mechanical cues may also play a fundamental role in dictating how cells explore the surrounding space. Haptotaxis (i.e., directed motion driven by gradients in the local density of adhesion sites) and durotaxis (i.e., directed motion driven by gradients in the stiffness of the surrounding extracellular matrix) are well-studied examples of taxa driven by mechanical cues [3-5].

In vivo, cells crawl through topographically intricate environments, such as the extracellular matrix, blood and lymphatic vessels, other cells, etc., that can significantly influence migration strategies [6-13]. A striking manifestation of this phenomenon, termed "topotaxis," has been reported by Park et al. in the context of melanoma cells on a substrate featuring a spatially varying density of nanosized posts [14]. Depending on the effective stiffness of the cortical cytoskeleton, cells have been observed to migrate toward regions of either higher or lower post density, as a result of the interplay between two simultaneous signaling pathways activated by the extracellular matrix input. Analogously, adhesive ratchets [15-17] and several types of anisotropic topographical features at the

\footnotetext{
*Corresponding author: giomi@lorentz.leidenuniv.nl
}

subcellular scale have also been shown to lead to directed cell migration [15,18-21]. A different manifestation of topotaxis has been recently demonstrated by Wondergem et al. using a spatial gradient in the density of cell-sized topographical features [22]. In these experiments, highly motile, persistently migrating cells (i.e., cells performing amoeboid migration) move on a substrate in between microfabricated pillars that act as obstacles and consequently force the cells to move around them. If the obstacles' density smoothly varies across the substrate, the cells have been shown to migrate toward the regions of lower obstacle density.

Unlike in the case of the topotactic behavior investigated by Park et al. in melanoma cell lines [14], the precise biophysical or biochemical principles behind the topographical guidance arising from cell-sized obstacles [22] are presently unknown. Yet, its occurrence for cells performing amoeboid migration suggests the possibility of cell-type-independent mechanisms that, separately from the cell's mechanosensing machinery, provide a generic route to the emergence of topotaxis at the large scale. In this article, we explore this hypothesis. Using numerical simulations and analytical arguments, we demonstrate that large-scale topotaxis can be mimicked by active Brownian particles (ABPs) constrained to move within an obstacle lattice characterized by a spatially varying density of particle-sized features. In this case, directed motion originates solely from the spatial modulation of persistence resulting from the interaction between the particles and the obstacles.

ABPs represent a simple stochastic model for selfpropelled particles, such as active Janus particles [23], and for cell motility on flat substrates [24]. ABPs perform persistent self-propelled motion in the direction of the particle orientation in combination with rotational diffusion of this 
orientation. The motion of active particles has been explored in several complex geometries, including convex [25,26] and nonconvex [27] confinements, mazes [28], walls of funnels [29], interactions with asymmetric [30,31] and chiral [32] passive objects, periodic [33] and random [34-37] obstacle lattices, and porous topographies [38]. For a review, see Refs. [39,40]. Because of the nonequilibrium nature of active particles, local asymmetries in the environment can be leveraged to create a drift; these particles have been demonstrated to perform chemotaxis [41,42], durotaxis [43], and phototaxis [44]. Furthermore, topographical cues, such as those obtained in the presence of arrays of asymmetric posts $[45,46]$ and ratchets consisting of asymmetric potentials [47-49] or asymmetric channels [50-53], have been shown to produce a directional bias in the motion of active particles reminiscent of those observed for cells. Finally, we stress that the topotactic behavior analyzed here results from gradients in particle-sized substrate features, and thus has no connection to the examples of topotaxis reported by Park et al. [14] which originates from topographical features at the subcellular scale. Furthermore, while inspired by experiments on amoeboid cell migration, the goal of our analysis is not to deliver a predictive theory that could be used in comparison with experiments on migrating cells, but merely to illustrate how large-scale topotaxis emerges in a toy model of active Brownian particles.

The paper is organized as follows: in Sec. II, we present our model for ABPs and their interaction with obstacles. In Sec. III A, we show that in the presence of a gradient in the obstacle density, ABPs drift, on average, in the direction of lower density. The speed of this net drift, here referred to as topotactic velocity, increases as a function of both the density gradient and the persistence length of the ABPs. In Sec. III B (numerically) and Sec. IIIC (analytically), we study ABPs in regular obstacle lattices and demonstrate that the origin of topotaxis of active particles can be found in the altered persistence length of the particles in the presence of obstacles.

\section{THE MODEL}

Our model of ABPs consists of disks of radius $R_{p}$ selfpropelling at constant speed $v_{0}$ along the unit vector $\boldsymbol{p}=$ $(\cos \theta, \sin \theta)$ and subject to rotational white noise. The dynamics of the particles is governed by the following overdamped equations:

$$
\begin{aligned}
& \frac{d \boldsymbol{r}}{d t}=v_{0} \boldsymbol{p}+\mu \boldsymbol{F}, \\
& \frac{d \theta}{d t}=\sqrt{2 D_{r}} \xi,
\end{aligned}
$$

where $\boldsymbol{r}=\boldsymbol{r}(t)$ is the position of the particle, $t$ is time, and $\mu$ is a mobility coefficient. The force $\boldsymbol{F}=\boldsymbol{F}(\boldsymbol{r})$ embodies the interactions between the particles and the obstacles. $\xi=\xi(t)$ is a random variable with zero mean, i.e., $\langle\xi(t)\rangle=0$, and time correlation $\left\langle\xi(t) \xi\left(t^{\prime}\right)\right\rangle=\delta\left(t-t^{\prime}\right)$. The extent of rotational diffusion is quantified by the rotational diffusion coefficient $D_{r}$, whereas translational diffusion is neglected under the assumption of large Péclet number: Pe $\gg 1$. Overall, this setup provides a reasonable toy model for highly motile cells such as those used in experimental studies of large-scale topotaxis $[22,54,55]$. For a study on the influence of the Péclet number on the motion of ABPs around obstacles, see, for example, Refs. [33,35].

In free space (i.e., $\boldsymbol{F}=\mathbf{0}$ ), ABPs described by Eqs. (1) perform a persistent random walk (PRW) with mean displacement $\langle\Delta \boldsymbol{r}(t)\rangle=0$ and mean squared displacement,

$$
\left\langle|\Delta \boldsymbol{r}(t)|^{2}\right\rangle=2 v_{0}^{2} \tau_{p}^{2}\left(\frac{t}{\tau_{p}}+e^{-t / \tau_{p}}-1\right),
$$

where $\Delta \boldsymbol{r}(t)=\boldsymbol{r}(t)-\boldsymbol{r}(0)$ and $\langle\cdot\rangle$ represents an average over $\xi$ (see, e.g., Ref. [40]). The constant $\tau_{p}=1 / D_{r}$, commonly referred to as persistence time, quantifies the typical timescale over which a particle tends to move along the same direction. Thus, over timescales shorter than the persistence time, $t \ll \tau_{p}$, ABPs move ballistically with speed $v_{0}$, i.e., $\left\langle|\Delta \boldsymbol{r}(t)|^{2}\right\rangle \approx\left(v_{0} t\right)^{2}$, while over timescales larger than the persistence time, $t \gg \tau_{p}$, ABPs diffuse, i.e., $\left\langle|\Delta \boldsymbol{r}(t)|^{2}\right\rangle=4 D t$, with $D=v_{0}^{2} \tau_{p} / 2$ the diffusion coefficient. From $\tau_{p}$, one can define a persistence length, $l_{p}=v_{0} \tau_{p}$, as the typical distance traveled by a particle before losing memory of its previous orientation. Consistently, the autocorrelation function of the velocity $\boldsymbol{v}=d \boldsymbol{r} / d t\left(\boldsymbol{v}=v_{0} \boldsymbol{p}\right.$ in free space $)$ is given by

$$
\langle\boldsymbol{v}(t+\Delta t) \cdot \boldsymbol{v}(t)\rangle=v_{0}^{2} e^{-\Delta t / \tau_{p}}
$$

Our ABPs roam within a two-dimensional array of circular obstacles of radius $R_{o}$. Following Refs. [26,27], the interactions between particles and obstacles are modeled via a force of the form

$$
\boldsymbol{F}= \begin{cases}-\frac{v_{0}}{\mu}(\boldsymbol{p} \cdot \boldsymbol{N}) \boldsymbol{N} & \text { if } \Delta \boldsymbol{r}_{o} \leqslant R \\ \mathbf{0} & \text { otherwise }\end{cases}
$$

where $\boldsymbol{N}$ is a unit vector normal to the obstacle surface, $\left|\Delta \boldsymbol{r}_{o}\right|$ is the distance between the obstacle center and the particle center, and the effective obstacle radius $R$ is the sum of the obstacle and the particle radii: $R=R_{o}+R_{p}$. Equation (4) describes a frictionless hard wall force that cancels the velocity component normal to the obstacle surface whenever the particle would penetrate the obstacle, and vanishes otherwise. Therefore, the obstacle force $\boldsymbol{F}$ is either repulsive or zero, but never attractive. We stress that the wall force does not influence the particle orientation $\boldsymbol{p}$. Thus, a particle slides along an obstacle until either the obstacle wall becomes tangential to $\boldsymbol{p}$ or rotational diffusion causes the particle to rotate away. This is consistent with experimental observations on self-propelled colloids [25] as well as various types of cells [56,57]. For details of the numerical implementation of Eqs. (1) and (4), see Appendix A. In the following sections, we measure time in units of the persistence time, i.e., $\tilde{t}=t / \tau_{p}$, and length in units of the effective obstacle radius, i.e., $\tilde{\ell}=\ell / R$.

\section{RESULTS}

The motion of ABPs in different lattices of obstacles is visualized in Fig. 1. Each panel shows 20 simulated trajectories with persistence length $\tilde{l}_{p}=5$. Figures 1 (a) and 1(b) show regular square lattices with dimensionless center-to-center obstacle spacings of $\tilde{d}=2.5$ and $\tilde{d}=4$, respectively. In Fig. 1, the obstacles are graphically represented as disks of radius $R$ and the ABPs as point particles. To avoid biasing the statistics of the particle trajectories, ABPs start at a random location inside the unit cell of the regular square lattice [Fig. 1(c)] at 

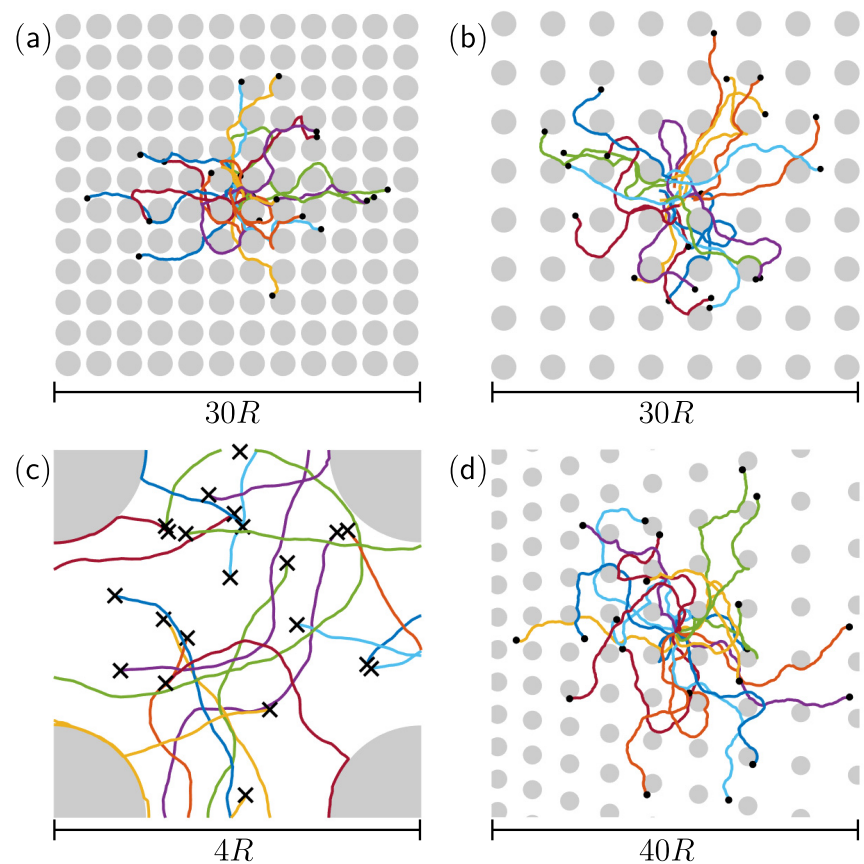

FIG. 1. Simulated trajectories of 20 active Brownian particles (ABPs) with persistence length $\tilde{l}_{p}=v_{0} \tau_{p} / R=5$ in different lattices of obstacles. The obstacles are graphically represented as disks of radius $R$ and the ABPs as point particles. (a),(b) ABPs in regular square lattices of obstacles with center-to-center obstacle spacings $\tilde{d}=d / R=2.5$ and $\tilde{d}=4$, respectively. The particles start at $\tilde{t}=0$ at a random location in the unit cell of the lattice and are simulated for a total time of $\tilde{t}=3$. (c) The unit cell of the regular square lattice showing the starting points (crosses) of the 20 trajectories in (b). (d) ABPs in a lattice with a linear gradient in obstacle spacing, quantified by a dimensionless parameter $r=0.07$ (see Appendix B), and $\tilde{d}=5$ at the center of the gradient region. The particles start at $\tilde{t}=0$ in the origin and are simulated for a total time of $\tilde{t}=5$.

$\tilde{t}=0$ with random orientation. All trajectories are shown for a total time of $\tilde{t}=3$. Comparing the spreading of the active particles in Fig. 1(a) with that in Fig. 1(b), we observe that the more dense the obstacle lattice is, the more it hinders the diffusion of the active particles. We will quantify this later.

To study topotaxis, we define an irregular square lattice comprising a linear gradient of the obstacle spacing in the positive $x$ direction. The latter is quantified in terms of a dimensionless parameter $r$ representing the rate at which the obstacle spacing decreases as $x$ increases. Thus $r=0$ corresponds to a regular square lattice, whereas large $r$ values correspond to rapidly decreasing obstacle spacing. Figure 1(d) shows this lattice for $r=0.07$ with 20 particle trajectories, starting in the origin at $\tilde{t}=0$ with a random orientation, plotted for a simulation time of $\tilde{t}=5$, where $\tilde{d}=5$ represents the obstacle spacing in the center of the gradient region. The gradient region has a finite width [not visible in Fig. 1(d)] and is flanked by regular square lattices to the left, with lattice spacing $\tilde{d}_{\text {min }}=2.1$, and to the right, with lattice spacing $\tilde{d}_{\max }=2 \tilde{d}-\tilde{d}_{\min }$. The minimal and maximal obstacle-toobstacle distances ( $\tilde{d}_{\min }$ and $\tilde{d}_{\text {max }}$, respectively) do not depend on the steepness of the gradient, and consequently the width of the gradient region decreases for steeper gradients (larger
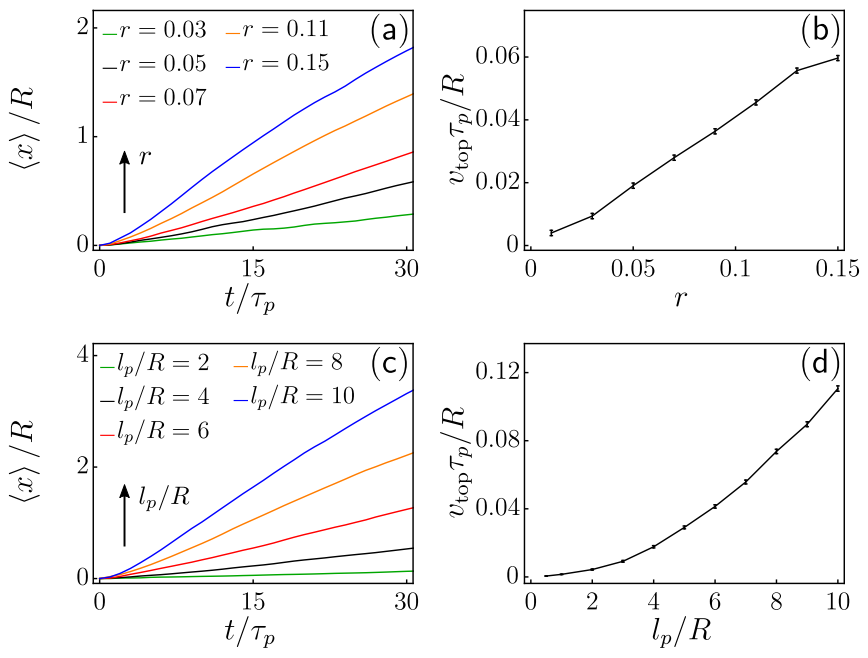

FIG. 2. The emergence of topotaxis in density gradient lattices. (a) $\langle\tilde{x}\rangle=\langle x\rangle / R$ as a function of time $\tilde{t}=t / \tau_{p}$ for several values of the density gradient $r$, with $\tilde{d}=d / R=5$ and $\tilde{l}_{p}=v_{0} \tau_{p} / R=5$. (b) Topotactic velocity, defined in the main text, in the $x$ direction as a function of the density gradient $r$ based on the data in (a). (c) $\langle\tilde{x}\rangle=\langle x\rangle / R$ as a function of time $\tilde{t}=t / \tau_{p}$ for several values of the persistence length $\tilde{l}_{p}=v_{0} \tau_{p} / R$, with $\tilde{d}=d / R=5$ and $r=$ 0.07 . (d) Topotactic velocity in the $x$ direction as a function of the persistence length $\tilde{l}_{p}$ based on the data in (c). Data in (a) and (c) represent averages over $10^{6}$ particles. Error bars in (b) and (d) are given by the standard error of $\langle x\rangle(t) /(R t)$ at $t=30 \tau_{p}$.

$r$ ). For a detailed description of both the regular and gradient lattices as well as an image of the gradient lattice including the regular lattices on the left and right, see Appendix B.

\section{A. The emergence of topotaxis}

To quantify topotaxis, we measure the average $x$ and $y$ coordinates, $\langle\tilde{x}\rangle$ and $\langle\tilde{y}\rangle$, as a function of time for $10^{6}$ particles. The results are given for five values of the dimensionless density gradient $r$ in Figs. 2(a) and 7(a) (Appendix C) for $x$ and $y$, respectively. The emergence of topotaxis is clear from Fig. 2(a): the active particles move, on average, in the positive $x$ direction, and hence in the direction of lower obstacle density. As expected by the symmetry of the lattice, there is no net motion in the $y$ direction independently of the value of $r$ [Fig. 7(a), Appendix C]. To further quantify topotaxis, we define the topotactic velocity as the average velocity in the positive $x$ direction in a time interval $\Delta t, v_{\text {top }}=\langle\Delta x\rangle / \Delta t$, and evaluate it between $\tilde{t}=0$ and $\tilde{t}=30$. Figures 2(a) and 2(b) show that $\tilde{v}_{\text {top }}$ is approximatively constant in time and proportional to the density gradient $r$.

Next, we investigate the effect of the intrinsic motion of the ABPs on topotaxis. This intrinsic motion is characterized by the persistence length $l_{p}=v_{0} \tau_{p}$, which uniquely determines the statistics of the particle trajectory in free space. Namely, if two types of ABPs have different $v_{0}$ and $\tau_{p}$, but the same $l_{p}$, their trajectories have the same statistical properties, even though faster particles move along these trajectories in a shorter time. Figure 2(c) shows $\langle\tilde{x}\rangle$ as a function of time for five values of $\tilde{l}_{p}$. The speed of topotaxis is again approximately constant in time and increases with $\tilde{l}_{p}$ [Fig. 2(d)]. 

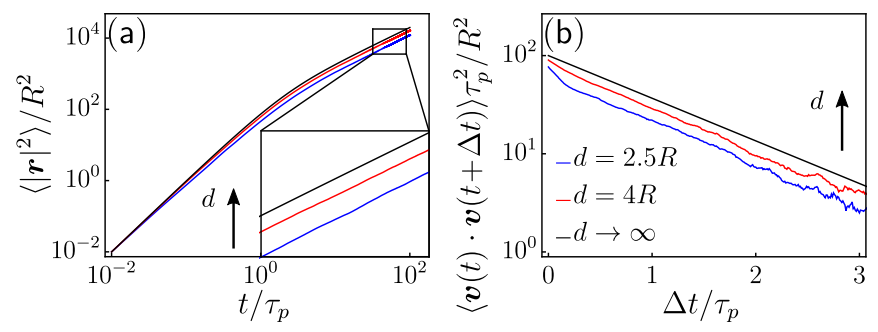

FIG. 3. Regular square lattices of obstacles modify the effective parameters of the persistent random walk. (a) Dimensionless mean square displacement $\left\langle|\Delta \tilde{\boldsymbol{r}}|^{2}\right\rangle=\left\langle|\Delta \boldsymbol{r}|^{2}\right\rangle / R^{2}$ as a function of dimensionless time $\tilde{t}=t / \tau_{p}$ for $\tilde{l}_{p}=v_{0} \tau_{p} / R=10$ in free space $(d \rightarrow \infty$, black line) and in the presence of square lattices with obstacle spacings $\tilde{d}=d / R=2.5$ (blue) and $\tilde{d}=4$ (red). The effective diffusion coefficient $D_{\text {eff }}$ is obtained from a linear fit to the long-time $\left(t>\tau_{p}\right)$ regime of the log-log data. (b) Dimensionless velocity autocorrelation function $\langle\tilde{\boldsymbol{v}}(t+\Delta t) \cdot \tilde{\boldsymbol{v}}(t)\rangle=\langle\boldsymbol{v}(t+\Delta t) \cdot \boldsymbol{v}(t)\rangle \tau_{p}^{2} / R^{2}$ as a function of $\Delta \tilde{t}=\Delta t / \tau_{p}$ for $\tilde{l}_{p}=v_{0} \tau_{p} / R=10$ in free space $(d \rightarrow$ $\infty)$ and in the presence of square lattices with obstacle spacings $\tilde{d}=$ 2.5 and $\tilde{d}=4$. The effective velocity $v_{\text {eff }}$ and effective persistence time $\tau_{\text {eff }}$ are obtained from a exponential fit to the autocorrelation function. MSD and VACF data represent averages over $10^{4}$ particles.

This trend partly results from the fact that increasing the persistence length corresponds either to an increment in $v_{0}$ or $\tau_{p}$, both resulting into an increase of $\tilde{v}_{0}$. However, Fig. 2(d) shows that $\tilde{v}_{\text {top }}$ increases faster than linear as a function of $\tilde{l}_{p}$, suggesting an additional effect caused by the obstacle lattice. As we will see in Sec. IIIB, this effect is caused by the fact that the lattice hinders ABPs with large persistence lengths more than ABPs with smaller persistence lengths. Finally, we note that there is no net motion in the $y$ direction irrespective of the persistence length, as expected by symmetry [Fig. 7(b), Appendix C].

\section{B. The physical origin of topotaxis}

The observed occurrence of topotaxis of ABPs is intuitive, as particles migrate in the direction where there is more available space. However, the mechanism by which ABPs are guided toward the less crowded regions is not obvious from the results in Sec. III A. To gain more insight into the physical origin of topotaxis, we investigate how particle motility depends on the local obstacle spacing. In doing so, we take inspiration from recent works $[43,58,59]$ that have shown, in the context of durotaxis, that persistent random walkers, moving in a spatial gradient of a position-dependent persistence length, show an average drift toward the region with larger persistence. As is the case in our system (Sec. III A), this effect is stronger in the presence of larger gradients [43,58]. In order to understand whether or not such a space-dependent persistence might explain the observed topotactic motion, we study and characterize the motion of ABPs in regular square lattices. To do so, we measure the mean squared displacement (MSD) $\left\langle|\Delta \boldsymbol{r}(t)|^{2}\right\rangle$ as a function of time and the velocity autocorrelation function (VACF) $\langle\boldsymbol{v}(t+\Delta t) \cdot \boldsymbol{v}(t)\rangle$ as a function of the time interval $\Delta t$ for $10^{4}$ particles for various lattice spacings $\tilde{d}$ and persistence lengths $\tilde{l}_{p}$.
Figure 3(a) shows a log-log plot of the MSD for $\tilde{l}_{p}=10$. The curve with $\tilde{d} \rightarrow \infty$ (black) represents the theoretical MSD in free space [Eq. (2)] and exhibits the well-known crossover from the ballistic regime (slope equal to 2 ) to the diffusive regime (slope equal to 1$)$ around $\tilde{t}=1\left(t=\tau_{p}\right)$. The hindrance of the obstacles is evident from the data obtained in regular lattices with $\tilde{d}=4$ (red curve) and $\tilde{d}=2.5$ (blue curve), as the MSD is smaller than the MSD in free space at all times (see, also, the inset). Moreover, the MSD is smaller for the smaller lattice spacing, as we already observed qualitatively in Figs. 1(a) and 1(b). The hindrance also manifests itself in the short-time $\left(t<\tau_{p}\right)$ regime, where the slope of the red and blue curves is slightly smaller than that of the black curve. When not interacting with obstacles, individual ABPs still move ballistically, but interactions with obstacles prevent them from moving along straight lines, thus enhancing the tendency to turn, which, in free space, originates solely from rotational diffusion.

The slope of the curves at timescales larger than the persistence time, on the other hand, is independent of the presence of obstacles and equal to 1 (see inset). In other words, even though the motion of the ABPs is hindered by the obstacles at all timescales, the long-time motion remains diffusive, as was also observed for ABPs in random obstacle lattices of low density [35]. Fitting the MSD at long times allows one to define an effective diffusion coefficient $D_{\text {eff }}$, namely,

$$
\left\langle|\Delta \boldsymbol{r}(t)|^{2}\right\rangle \underset{t \gg \tau_{p}}{\longrightarrow} 4 D_{\mathrm{eff}} t .
$$

Figure 3(b) shows the velocity autocorrelation function (VACF) on a semilogarithmic plot as a function of the time interval $\Delta \tilde{t}=\Delta t / \tau_{p}$ for $\tilde{l}_{p}=10$. The $d \rightarrow \infty$ curve again represents the theoretical curve in free space and shows exponential decay [Eq. (3)]. Interestingly, in the presence of increasing obstacle densities, and hence for smaller lattice spacings $\tilde{d}$, the velocity autocorrelation decreases but remains, to good approximation, exponential. From this numerical evidence, we conclude that the average motion of ABPs in a two-dimensional square lattice can be described as a persistent random walk with an effective velocity $v_{\text {eff }}$ and an effective persistence time $\tau_{\text {eff }}[35]$,

$$
\langle\boldsymbol{v}(t+\Delta t) \cdot \boldsymbol{v}(t)\rangle=v_{\mathrm{eff}}^{2} e^{-\Delta t / \tau_{\mathrm{eff}}} .
$$

Figure 4 shows $D_{\text {eff }}, \tau_{\text {eff }}$, and $v_{\text {eff }}$, normalized by their free space values, as a function of the obstacle spacing $\tilde{d}$ for three values of the free space persistence length $\tilde{l}_{p}$. Starting with the effective diffusion coefficient [Fig. 4(a)], we observe that for every value of the persistence length, the effective diffusion coefficient $D_{\text {eff }}$ increases as a function of $\tilde{d}$ until it approaches the free space diffusion coefficient $D$ for large $\tilde{d}$. This is consistent with what we observed in Figs. 1(a), 1(b), and 3: ABPs on low density lattices spread out more than ABPs on high density lattices. Moreover, the effective diffusion coefficient deviates more from its free space value for large $\tilde{l}_{p}$ than it does for small $\tilde{l}_{p}$. This is intuitive because more persistent particles tend to move longer along the same direction and therefore are hindered more in their motion by the obstacle lattice.

The effective persistence time $\tau_{\text {eff }}$ [Fig. 4(b)] and the effective velocity $v_{\text {eff }}$ [Fig. 4(c)], both extracted from the velocity autocorrelation function [Eq. (6)], show a similar 

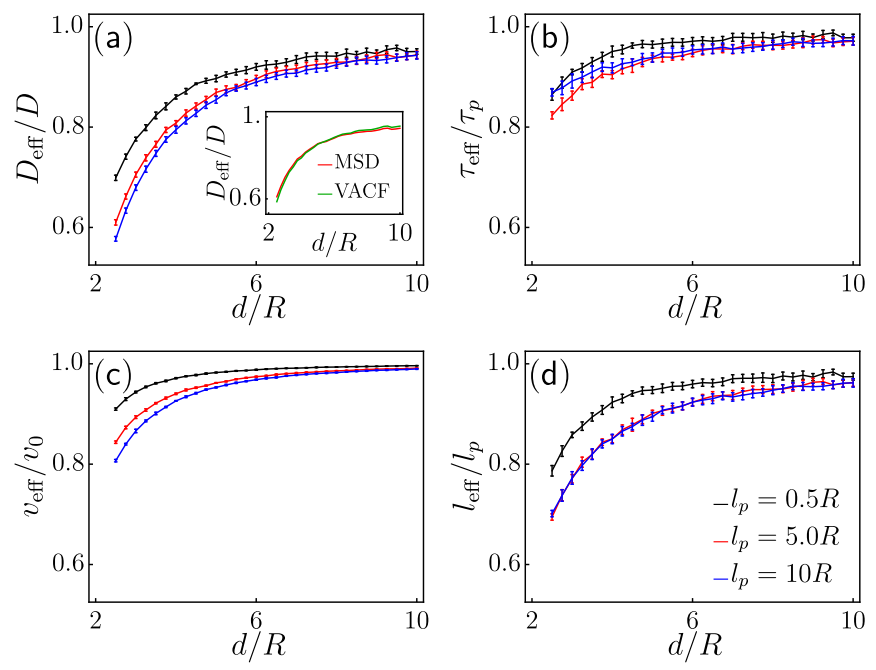

FIG. 4. Effective parameters of the persistent random walk in regular lattices of obstacles. (a) Normalized effective diffusion coefficient $D_{\text {eff }} / D$, obtained from the mean squared displacement [Eq. (5)], as a function of the normalized obstacle spacing $\tilde{d}=d / R$ for three values of the normalized persistence length $\tilde{l}_{p}=v_{0} \tau_{p} / R$. Inset shows $D_{\text {eff }}$ for $\tilde{l}_{p}=5$ obtained via the mean squared displacement (MSD) and via the velocity autocorrelation function (VACF), using $D_{\text {eff }}=v_{\text {eff }}^{2} \tau_{\text {eff }} / 2$. (b) Normalized effective persistence time $\tau_{\text {eff }} / \tau_{p}$, (c) normalized effective velocity $v_{\text {eff }} / v_{0}$, both obtained from the velocity autocorrelation function [Eq. (6)], and (d) normalized effective persistence length $l_{\text {eff }} / l_{p}=v_{\text {eff }} \tau_{\text {eff }} /\left(v_{0} \tau_{p}\right)$ as functions of the normalized obstacle spacing $\tilde{d}=d / R$ for three values of the normalized persistence length $\tilde{l}_{p}=v_{0} \tau_{p} / R$. Data points represent the average of 10 independent measurements from MSD or VACF data (Fig. 3). The error bars show the corresponding standard deviations.

trend: they increase as a function of $\tilde{d}$ until they approach their free space values at high $\tilde{d}$, and they deviate more from their free space values for large $\tilde{l}_{p}$ than they do for small $\tilde{l}_{p}$. These data show that the obstacles cause the ABPs, on average, to move slower and turn more quickly. Despite the orientation $\boldsymbol{p}$ not being affected by the obstacles [see Eq. (4)], the latter force the particles to move tangentially to the wall, thus enhancing their tendency to turn. The decreased effective velocity, with respect to free space, is intuitive given the interactions between particles and obstacles [Eq. (4)], which slow down the ABPs. The decreased effective persistence time, on the other hand, is less obvious as one could imagine the periodic obstacle lattice to guide ABPs along straight lines, as reported in Refs. [33,60]. Apparently, this potential guiding mechanism is outcompeted in our system by the fact that encounters of ABPs with individual obstacles at shorter timescales cause them to change their direction of motion more quickly than in free space. In Sec. IIIC, we will study these short-timescale interactions in greater detail.

Combining the effective persistence time [Fig. 4(b)] and the effective velocity [Fig. 4(c)] gives the effective persistence length $l_{\text {eff }}=v_{\text {eff }} \tau_{\text {eff }}$ and the effective diffusion coefficient $D_{\text {eff }}=v_{\text {eff }}^{2} \tau_{\text {eff }} / 2$. The inset of Fig. 4(a) shows the effective diffusion coefficient for $\tilde{l}_{p}=5$, calculated both by using the effective persistence time and effective velocity from the velocity autocorrelation function (VACF) and by a direct measurement from the mean squared displacement (MSD). The excellent agreement between $D_{\text {eff }}$ measured at short and long timescales (using the VACF and MSD, respectively) is another indication that the motion of the ABPs in regular square obstacle lattices can indeed be considered to be an effective persistent random walk.

The effective persistence length $l_{\text {eff }}=v_{\text {eff }} \tau_{\text {eff }}$ is plotted in Fig. 4(d). As anticipated, the effective persistence length increases with increasing lattice spacing $\tilde{d}$, consistent with findings of ABPs in random obstacle lattices [35] and a model of persistently moving cells in a tissue of stationary cells [61]. This effect increases with the free space persistence length, as the motion of less persistent particles is randomized before they can reach an obstacle. Furthermore, the difference between data with $\tilde{l}_{p}=5$ and $\tilde{l}_{p}=10$ is negligible, indicating that the free space persistence length $\tilde{l}_{p}$ affects particle motion only when it is comparable with the lattice spacing.

These observations, combined with those in Refs. $[43,58,59]$ which demonstrate a net flux of persistent random walkers toward regions of larger persistence, ultimately explain the origin of topotaxis in our system. ABPs migrate, on average, toward regions of higher persistence, and hence to regions of lower obstacle density. Moreover, the dependence of the effective persistence length $l_{\text {eff }}$ on the free space persistence length $\tilde{l}_{p}$ [Fig. 4(d)] justifies the superlinear increase of the topotactic velocity $\tilde{v}_{\text {top }}$ as a function of $\tilde{l}_{p}$ [Fig. 2(d)]. In addition to the normal speed-up due to the higher persistence, more persistent particles experience a larger gradient in persistence.

\section{Fokker-Planck equation for regular lattices}

As we explained in Sec. III B, topotaxis in our model of ABPs crucially relies on the fact that even when trapped in an array of obstacles, ABPs still behave as persistent random walkers. The physical origin of this behavior is, however, less clear from the numerical simulations. In this section, we rationalize this observation using some simple analytical arguments. The probability distribution function $P=P(\boldsymbol{r}, \theta, t)$ of the position and orientation of an ABP, whose dynamics is governed by Eqs. (1), evolves in time based on the following Fokker-Planck equation:

$$
\frac{\partial P}{\partial t}=-v_{0} \boldsymbol{p} \cdot \nabla P-\mu \nabla \cdot(P \boldsymbol{F})+D_{R} \frac{\partial^{2} P}{\partial \theta^{2}},
$$

subject, at all times, to the normalization constraint,

$$
\int d \boldsymbol{r} d \theta P(\boldsymbol{r}, \theta, t)=1,
$$

with $d \boldsymbol{r}=d x d y$. Equation (7) cannot be solved exactly, but useful insights can be obtained by calculating the rate of change of the mean squared displacement $\left\langle|\Delta \boldsymbol{r}|^{2}\right\rangle$. Here we assume $\boldsymbol{r}(0)=\mathbf{0}$, which yields $|\Delta \boldsymbol{r}|^{2}=|\boldsymbol{r}|^{2}=x^{2}+y^{2}$, and

$$
\frac{\partial\left\langle|\boldsymbol{r}|^{2}\right\rangle}{\partial t}=\int d \boldsymbol{r} d \theta|\boldsymbol{r}|^{2} \frac{\partial P(\boldsymbol{r}, \theta, t)}{\partial t}
$$

Upon substituting Eq. (7) in Eq. (9) and integrating by parts, we obtain

$$
\frac{\partial\left\langle|\boldsymbol{r}|^{2}\right\rangle}{\partial t}=2 v_{0}\langle\boldsymbol{r} \cdot \boldsymbol{p}\rangle+2 \mu\langle\boldsymbol{r} \cdot \boldsymbol{F}\rangle .
$$


Analogously, the term $\langle\boldsymbol{r} \cdot \boldsymbol{p}\rangle$ evolves accordingly to

$$
\frac{\partial\langle\boldsymbol{r} \cdot \boldsymbol{p}\rangle}{\partial t}=v_{0}-D_{R}\langle\boldsymbol{r} \cdot \boldsymbol{p}\rangle+\mu\langle\boldsymbol{p} \cdot \boldsymbol{F}\rangle .
$$

In free space $(\boldsymbol{F}=\mathbf{0})$, Eqs. (10) and (11) can be solved exactly, using the boundary condition $\boldsymbol{r}(t=0)=\mathbf{0}$, to find

$$
\langle\boldsymbol{r} \cdot \boldsymbol{p}\rangle_{\boldsymbol{F}=\mathbf{0}}=\frac{v_{0}}{D_{R}}\left(1-e^{-D_{R} t}\right),
$$

and the mean squared displacement $\left\langle|\boldsymbol{r}|^{2}\right\rangle$ given by Eq. (2). A generic nonzero $\boldsymbol{F}$ compromises the closure of the equations, thus making the problem intractable with exact methods. Nevertheless, it is possible to use some simplifying assumptions to obtain intuitive results about $D_{\text {eff }}$ and $\tau_{\text {eff }}$ (Fig. 4) at short $\left(t \ll 1 / D_{r}=\tau_{p}\right)$ and long $\left(t \gg 1 / D_{r}=\tau_{p}\right)$ timescales.

At short timescales, we can assume a particle to be still relatively close to its initial position, $\boldsymbol{r}(0)=\mathbf{0}$. Thus one can expand the force in Eq. (11) at the linear order in $\boldsymbol{r}$, i.e., $\boldsymbol{F}(\boldsymbol{r}) \approx \boldsymbol{F}(\mathbf{0})+\nabla \boldsymbol{F}(\mathbf{0}) \cdot \boldsymbol{r}$. Evidently, such an expansion is ill defined for discontinuous forces such as that given by Eq. (4). However, one can imagine to smoothen the force (for instance, using a truncated Fourier expansion) without altering the qualitative picture. Under this approximation, the short-time motion is then analogous to that of ABPs confined by a harmonic trap (see, e.g., Refs. [62-65]). By the symmetry of the obstacle lattice, $\boldsymbol{F}(\mathbf{0})=\mathbf{0}, \partial_{y} F_{x}(\mathbf{0})=\partial_{x} F_{y}(\mathbf{0})=0$, and the constant $\partial_{x} F_{x}(\mathbf{0})=\partial_{y} F_{y}(\mathbf{0})<0$, as the horizontal (vertical) component of the force experienced by a particle moving in the positive $x$ direction ( $y$ direction), becomes more negative as the particle moves away from the origin. The approximation allows us to write

$$
\left.\langle\boldsymbol{p} \cdot \boldsymbol{F}\rangle\right|_{t \ll \tau_{p}}=\frac{\partial F_{x}}{\partial x}(\mathbf{0})\langle\boldsymbol{r} \cdot \boldsymbol{p}\rangle,
$$

and by inserting Eq. (13) into Eq. (11), we find

$$
\left.\frac{\partial\langle\boldsymbol{r} \cdot \boldsymbol{p}\rangle}{\partial t}\right|_{t \ll \tau_{p}}=v_{0}-\left[D_{r}-\mu \frac{\partial F_{x}}{\partial x}(\mathbf{0})\right]\langle\boldsymbol{r} \cdot \boldsymbol{p}\rangle .
$$

Solving Eq. (14) yields

$$
\left.\langle\boldsymbol{r} \cdot \boldsymbol{p}\rangle\right|_{t \ll \tau_{p}}=\frac{v_{0}}{D_{r, \text { eff }}}\left(1-e^{-D_{r, \text { eff }} t}\right),
$$

with $D_{r, \text { eff }}=D_{r}-\mu \partial_{x} F_{x}(\mathbf{0})>D_{r}$. A plot of $\langle\boldsymbol{r} \cdot \boldsymbol{p}\rangle$ versus $\Delta t / \tau_{p}$, obtained from our numerical simulations, is shown in Fig. 5, demonstrating that despite the force given in Eq. (4) being discontinuous, the trend entailed in Eq. (15) is preserved. By comparing Eq. (15) with its free space equivalent [Eq. (12)], we identify $D_{r \text {,eff }}$ as an increased effective rotational diffusion coefficient. This implies a decreased effective persistence time, consistent with the data in Fig. 4(b). The above analysis shows that, to first order, the observed decrease in effective persistence time simply results from the short-time interactions, within a unit cell of the lattice, that cause the particles to turn more frequently than in free space. Finally, substituting Eq. (15) in Eq. (10) and taking again the first-order Taylor expansion for $\boldsymbol{F}$ allows one to solve Eq. (10) exactly. Expanding this exact solution at the second order in time yields

$$
\left.\left\langle|\boldsymbol{r}|^{2}\right\rangle\right|_{t \ll \tau_{p}}=v_{0}^{2} t^{2},
$$

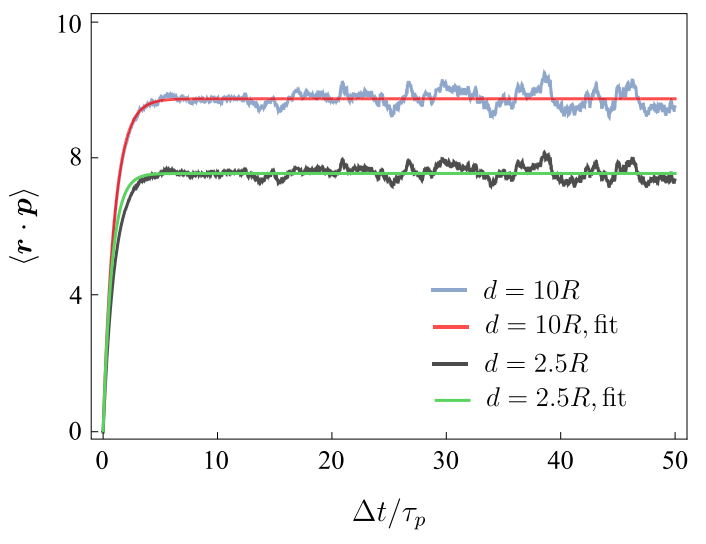

FIG. 5. The correlation function $\langle\boldsymbol{r} \cdot \boldsymbol{p}\rangle$ vs $\Delta t / \tau_{p}$ obtained from a numerical integration of Eqs. (1) in a regular obstacle lattice for different values of lattice spacings, namely, $\tilde{d}=d / R=2.5$ (black) and $\tilde{d}=10$ (blue). The green (red) lines show the fit of the data for $\tilde{d}=2.5(\tilde{d}=10)$ using Eq. (15). We find that the numerical data from our model match qualitatively with the analytical expression of Eq. (15) at short times, and on average plateaus to a constant value at long times, consistent with our analytical predictions in obtaining Eq. (17). From the analytical fits, we find $D_{r \text {,eff }}=$ $1.02847 D_{r}\left(D_{r \text {,eff }}=1.32592 D_{r}\right)$ for $\tilde{d}=10(\tilde{d}=2.5)$. This verifies that $D_{r \text {,eff }}(\tilde{d}=2.5)>D_{r \text {,eff }}(\tilde{d}=10)>D_{r}$ (free space $)$, i.e., the effective rotational diffusion coefficient increases with an increase in obstacle density.

which is the standard ballistic regime of the mean squared displacement. Hence, the decreased effective velocity observed in Fig. 4(c) originates from interactions with obstacles at larger timescales $\left[t \sim \tau_{p}\right.$; see, also, Fig. 3(b)].

In the long timescale $\left(t \gg 1 / D_{r}=\tau_{p}\right)$, the particles reach a diffusive steady state, and thus $\partial_{t}\langle\boldsymbol{r} \cdot \boldsymbol{p}\rangle=0$ (see Fig. 5 for $\Delta t / \tau_{p} \gg 1$ ). Hence, solving Eq. (11) for $\langle\boldsymbol{r} \cdot \boldsymbol{p}\rangle$ and substituting in Eq. (10) yields

$$
\left.\frac{\partial\left\langle|\boldsymbol{r}|^{2}\right\rangle}{\partial t}\right|_{t \gg \tau_{p}}=\frac{2 v_{0}^{2}}{D_{r}}\left(1+\frac{\mu}{v_{0}}\langle\boldsymbol{p} \cdot \boldsymbol{F}\rangle+\frac{\mu D_{r}}{v_{0}^{2}}\langle\boldsymbol{r} \cdot \boldsymbol{F}\rangle\right) .
$$

As the long-time behavior is diffusive, the expression on the right-hand side of Eq. (17) is constant and equal to $4 D_{\text {eff }}$. Now, according to Eq. (4), $\mu(\boldsymbol{p} \cdot \boldsymbol{F})=\mathbf{0}$ if $\left|\Delta \boldsymbol{r}_{o}\right|>R$, and $\mu(\boldsymbol{p} \cdot \boldsymbol{F})=-v_{0}(\boldsymbol{p} \cdot \boldsymbol{N})^{2}$ otherwise. Thus, $\langle\boldsymbol{p} \cdot \boldsymbol{F}\rangle<0$. This term shows that diffusion is slowed down because the obstacle force $F$ always slows down the particles (but never accelerates them). Moreover, for more dense obstacle lattices, particles interact with obstacles more often, which explains the observed dependence of $D_{\text {eff }}$ on the obstacle spacing in Fig. 4(a). Analogously, since particles move in an open space and, on average, away from the center, $\langle\boldsymbol{r} \cdot \boldsymbol{F}\rangle<0$ (i.e., the repulsion forces due to the obstacles are directed more often toward the origin than toward infinity, further slowing down diffusion). Thus $D_{\text {eff }}<D$, consistent with our numerical simulations [Figs. 3 and $4(a)$ ].

Finally, in the presence of very steep gradients in the obstacle density, when ABPs are unable to sample a large number of unit cells before experiencing a change in obstacle density, we expect the long-time behavior of the particles, 
hence $v_{\text {eff }}$, to change compared to the simple picture presented here, possibly affecting the topotactic mechanism.

\section{DISCUSSION AND CONCLUSIONS}

In this article, we investigated topotaxis, i.e., directed motion driven by topographical gradients, in a toy model of ABPs constrained to move within a two-dimensional array of obstacles of smoothly varying density. We found that ABPs migrate preferentially toward regions of lower density with a velocity that increases with the gradient in the lattice spacing and with the particles' persistence length. In our model, the origin of topotaxis crucially relies on the fact that even when moving in a lattice of obstacles, ABPs still behave as persistent random walkers, but with renormalized transport coefficients: $\tau_{\text {eff }}$ and $v_{\text {eff }}$. As these depend on the topography of the substrate, here quantified in terms of lattice spacing, topographical gradients result in spatially varying persistence in the motion of the particles, which in turn drives directed motion toward regions of larger persistence $[43,58,59]$. We note that the motion we report here, just like the durotactic motion described in Refs. [43,58], is perhaps better described as a "kinesis" than as a "taxis" because the underlying mechanism of transport is a nondirectional change in behavior induced by a purely positional cue. This is in contrast to the true directional bias underlying, for instance, the chemotaxis of E. coli [66] which leads to significantly more efficient transport [59].

Several questions remain open to future investigation. For instance, how is the picture affected by translational diffusion? Is topotaxis robust against competing directional cues, such as chemotaxis [22]? How sensitive is the performance of topotaxis with respect to the obstacles' shape $[25,45,46]$, the type of motion (e.g., persistent random walk, run-andtumble, Lévy walk, etc. [25,28,38,67-69]), and the details of particle-obstacle interactions [36,45,70-72]? For instance, one can expect that specific anisotropic shapes could be devised with the purpose of focusing the particles, thus tuning the topotactic behavior. Similarly, another interesting setting of the problem could be obtained by considering random arrangements of obstacles, where, unlike in the lattices studied here, particles can be trapped into convex-shaped features that can significantly alter their motion [35,38].

Finally, although here we demonstrated that topotaxis can be solely driven by the interplay between topographical gradients and persistent random motion, whether this is sufficient to explain large-scale topotaxis of cells remains an open problem. A quantitative comparison between our numerical data and experiments on highly motile cells [22] shows, in fact, discrepancies that could be ascribed to the enormously more complex interactions between cells and their environment. Specifically, the topotactic velocity in our simulations is of the order of $1 \%$ of the intrinsic particle speed (Fig. 2), whereas in the experiments on cells this ratio is approximately $5 \%$, provided that the obstacles are not spaced further apart than the cell size [22]. In order to better understand this surprising efficiency, the large-scale topotactic response of several types of persistently and individually moving cells, such as amoeba [73], invasive (amoeboid) cancer cells [74,75], or leukocytes [76], could be compared. On the theoretical side, we are currently addressing the problem using more biologically realistic models of cell motility based on the cellular Potts model [61,77], which allow explicitly taking into account effects such as the resistance of cells against deformations, adhesion between cells and obstacles, and more realistic cell-obstacle interactions.

\section{ACKNOWLEDGMENTS}

This work was supported by funds from the Netherlands Organization for Scientific Research (NWO/OCW), as part of the Frontiers of Nanoscience program (L.G.), the Netherlands Organization for Scientific Research (NWO-ENW) within the Innovational Research Incentives Scheme (R.M.H.M.; Vici 2017, Grant No. 865.17.004), and the Leiden/Huygens fellowship (K.S.).

\section{APPENDIX A: NUMERICAL METHODS}

We numerically generate particle trajectories that perform a persistent random walk by discretizing the equations of motion as follows [43]: a particle starts at position $\boldsymbol{r}_{0}$ at $t=0$, after which the particle is moved by a distance $v_{0} \Delta t$ in a random initial direction $-\pi<\theta_{1}<\pi$, such that the new position is $\boldsymbol{r}_{1}=\boldsymbol{r}_{0}+v_{0} \Delta t \boldsymbol{p}\left(\theta_{1}\right)$. For all subsequent time steps, the angle at time step $n, \theta_{n}$, is updated by adding a small deviation angle to the angle of the previous time step, $\theta_{n}=\theta_{n-1}+\delta \theta$. Here, $-\pi<\delta \theta<\pi$ is extracted randomly from a Gaussian distribution with mean 0 and variance $\sigma^{2}=$ $2 \Delta t / \tau_{p}$ using the Box-Muller transform. The new position of the particle, $\boldsymbol{r}_{n}$, is then found by $\boldsymbol{r}_{n}=\boldsymbol{r}_{n-1}+v_{0} \Delta t \boldsymbol{p}\left(\theta_{n}\right)$, with $\boldsymbol{r}_{n-1}$ the position at the previous time step.

If the update step moves the particle into an obstacle, however, the particle-obstacle force [Eq. (4)] is triggered. In that case, the normal component of the attempted displacement is subtracted, and the actual displacement is given by the tangential component of the attempted displacement, $\boldsymbol{r}_{n}=$ $\boldsymbol{r}_{n-1}+v_{0} \Delta t\left[\boldsymbol{p}\left(\theta_{n}\right) \cdot \boldsymbol{T}\right] \boldsymbol{T}$, with $\boldsymbol{T}$ the tangent unit vector of the obstacle surface at the point of the surface closest to $\boldsymbol{r}_{n-1}$. This procedure is implied by Euler integration of Eq. 1(a) with the force $\boldsymbol{F}$ described by Eq. (4). We choose the time step $\Delta t$ such that it is much smaller than the persistence time, $\Delta t \ll \tau_{p}$, and such that every displacement is much smaller than the obstacle radius, $v_{0} \Delta t \ll R$. In all reported simulations we have used $\Delta t=0.01 \tau_{p}$.

\section{APPENDIX B: OBSTACLE LATTICES}

We define a regular square lattice of obstacles with the coordinates of the centers of the obstacles given by

$$
\begin{aligned}
& x(n, m)=n d+\frac{d}{2}, \\
& y(n, m)=m d+\frac{d}{2},
\end{aligned}
$$

where $n, m \in \mathbb{Z}$ are the obstacle numbers and $d$ is the distance between the centers of two neighboring obstacles. The term $d / 2$ is added to make sure that the origin of the coordinate system is in the middle of four obstacles. An illustration of this lattice is given in Figs. 1(a) and 1(b). 


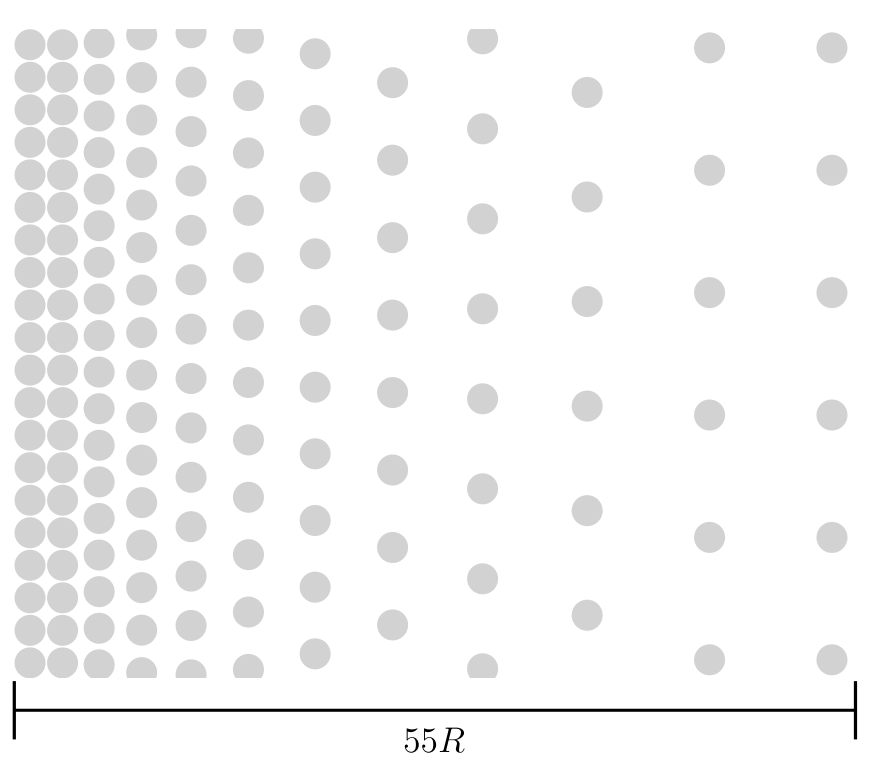

FIG. 6. Snapshot of the gradient lattice as described in Appendix B. The gradient region is characterized by $r=0.15$ and $\tilde{d}=d / R=5$. The obstacles are graphically represented as disks of radius $R$. The lattice spacing varies from $\tilde{d}_{\min }=2.1$ to $\tilde{d}_{\max }=7.9$ over the $x$ range $\left[x_{\min }, x_{\max }\right]=[-19,19]$. The gradient region is flanked by a regular square lattice with $\tilde{d}=\tilde{d}_{\min }$ on the left $\left(x<x_{\min }\right)$ and by a regular square lattice with $\tilde{d}=\tilde{d}_{\max }$ on the right $\left(x>x_{\max }\right)$. Only the first two columns of both (infinitely large) regular lattices are shown.

We define an irregular square lattice with a linear gradient of the obstacle spacing in the positive $x$ direction. The gradient region has a finite width, is centered in the origin, and is flanked by regular square lattices to the left and to the right. The coordinates of the centers of the obstacles in the gradient region are given by

$$
\begin{aligned}
& x(n, m)=\frac{d}{1-e^{-r}}\left(e^{r n}-1\right)+\frac{d}{2}, \\
& y(n, m)=d\left(m+\frac{1}{2}\right) e^{r n},
\end{aligned}
$$

where $n, m \in \mathbb{Z}$ are again the obstacle numbers, $d$ is the distance between the centers of obstacles with $(n, m)=(0,0)$ and $(n, m)=(-1,0)$ (i.e., the lattice spacing in the origin), and $r$ is a dimensionless number that quantifies the gradient in the obstacle spacing.

Equation (B2) represents an obstacle lattice where the lattice spacing depends exponentially on the horizontal obstacle number $n$, such that $x(n, m)-x(n-1, m)=d e^{r n}$ and $y(n, m)-y(n, m-1)=d e^{r n}$. This exponential gradient in the obstacle spacing, as a function of the obstacle number $n$, leads to a linear gradient in the obstacle spacing as a function of the horizontal coordinate $x$. This can be seen by calculating
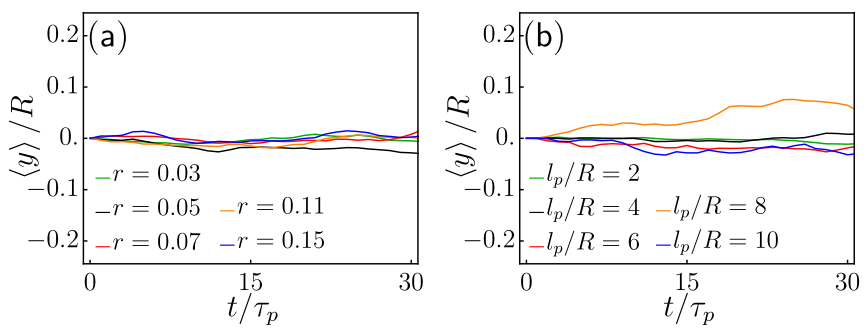

FIG. 7. There is no average drift in the $y$ direction in density gradient lattices. (a) $\langle\tilde{y}\rangle=\langle y\rangle / R$ as a function of time $\tilde{t}=t / \tau_{p}$ for five values of the density gradient $r$, with $\tilde{d}=d / R=5$ and $\tilde{l}_{p}=$ $v_{0} \tau_{p} / R=5$. (b) $\langle\tilde{y}\rangle=\langle y\rangle / R$ as a function of time $\tilde{t}=t / \tau_{p}$ for five values of the persistence length $\tilde{l}_{p}=v_{0} \tau_{p} / R$, with $\tilde{d}=d / R=5$ and $r=0.07$.

the difference in obstacle distance between two adjacent pairs of obstacles, divided by the distance between those two pairs,

$$
\frac{[x(n+1)-x(n)]-[x(n)-x(n-1)]}{x(n)-x(n-1)}=e^{r}-1,
$$

which is independent of $n$, as required for a linear gradient. In the limit of $r \rightarrow 0$, Eqs. (B2) reduce to the regular square lattice given in Eqs. (B1).

The gradient lattice is cut off on the left side at $x_{\min }<0$, where the vertical distance between two neighboring obstacles, $y(n, m)-y(n, m-1)$, would otherwise become smaller than a minimal distance $d_{\min }=2.1 R$. At the first column of obstacles for which this is the case, the vertical coordinates [Eq. (B2b)] are replaced by $y(n, m)=m d_{\text {min }}+d_{\min } / 2$. To the left of this transition column $\left(x<x_{\min }\right)$, a regular obstacle lattice with spacing $d_{\text {min }}$ is placed such that the transition column is part of this regular lattice.

On the right side, the gradient lattice is cut off at $x_{\max }=$ $-x_{\min }$. To the right of this cutoff $\left(x>x_{\max }\right)$, a regular obstacle lattice with spacing $d_{\max }=2 d-d_{\min }$ is placed such that the horizontal distance between the rightmost column of the gradient lattice and the leftmost column of the regular lattice is equal to $d_{\text {max }}$. Thus, the gradient lattice connects two regular square lattices of lattice spacings $d_{\min }$ and $d_{\max }$. The width of the gradient region, $2 x_{\max }$, then depends on the gradient parameter $r$. For an illustration of the gradient lattice for $r=0.15$ and $\tilde{d}=d / R=5$, see Fig. 6 .

\section{APPENDIX C: AVERAGE MOTION IN $y$}

We plot $\langle\tilde{y}\rangle(\tilde{t})$ of $10^{6}$ particles moving in a density gradient lattice with $\tilde{d}=5$, starting in the origin with a random orientation, for several values of the dimensionless density gradient $r$ in Fig. 7(a), and for several values of the persistence length $\tilde{l}_{p}$ in Fig. 7(b). As expected, there is no average drift in the $y$ direction. The fluctuations in $\langle y\rangle$ are of the order of $10 \%$ of the effective radius $R$.
[1] P. J. M. Van Haastert and P. N. Devreotes, Chemotaxis: Signaling the way forward, Nat. Rev. Mol. Cell Biol. 5, 626 (2004).
[2] K. F. Swaney, C.-H. Huang, and P. N. Devreotes, Eukaryotic chemotaxis: A network of signaling pathways controls motility, 
directional sensing, and polarity, Annu. Rev. Biophys. 39, 265 (2010).

[3] J. B. McCarthy and L. T. Furcht, Laminin and fibronectin promote the haptotactic migration of B16 mouse melanoma cells in vitro, J. Cell Biol. 98, 1474 (1984).

[4] C. M. Lo, H. B. Wang, M. Dembo, and Y. L. Wang, Cell movement is guided by the rigidity of the substrate, Biophys. J. 79, 144 (2000).

[5] G. Charras and E. Sahai, Physical influences of the extracellular environment on cell migration, Nat. Rev. Mol. Cell Biol. 15, 813 (2014).

[6] D.-H. Kim, K. Han, K. Gupta, K. W. Kwon, K.-Y. Suh, and A. Levchenko, Mechanosensitivity of fibroblast cell shape and movement to anisotropic substratum topography gradients, Biomaterials 30, 5433 (2009).

[7] D. Arcizet, S. Capito, M. Gorelashvili, C. Leonhardt, M. Vollmer, S. Youssef, S. Rappl, and D. Heinrich, Contactcontrolled amoeboid motility induces dynamic cell trapping in 3d-microstructured surfaces, Soft Matter 8, 1473 (2012).

[8] X. Sun, M. K. Driscoll, C. Guven, S. Das, C. A. Parent, J. T. Fourkas, and W. Losert, Asymmetric nanotopography biases cytoskeletal dynamics and promotes unidirectional cell guidance, Proc. Natl. Acad. Sci. 112, 12557 (2015).

[9] C. D. Paul, P. Mistriotis, and K. Konstantopoulos, Cancer cell motility: Lessons from migration in confined spaces, Nat. Rev. Cancer 17, 131 (2016).

[10] N. D. Bade, T. Xu, R. D. Kamien, R. K. Assoian, and K. J. Stebe, Gaussian curvature directs stress fiber orientation and cell migration, Biophys. J. 114, 1467 (2018).

[11] L. Pieuchot, J. Marteau, A. Guignandon, T. Dos Santos, I. Brigaud, P.-F. Chauvy, T. Cloatre, A. Ponche, T. Petithory, P. Rougerie, M. Vassaux, J.-L. Milan, N. Tusamda Wakhloo, A. Spangenberg, M. Bigerelle, and K. Anselme, Curvotaxis directs cell migration through cell-scale curvature landscapes, Nat. Commun. 9, 3995 (2018).

[12] D.-H. Kim, A. J. Ewald, J. Park Kshitiz, M. Kwak, R. S. Gray, C.-Y. Su, J. Seo, S. S. An, and A. Levchenko, Biomechanical interplay between anisotropic re-organization of cells and the surrounding matrix underlies transition to invasive cancer spread, Sci. Rep. 8, 14210 (2018).

[13] B. Winkler, I. S. Aranson, and F. Ziebert, Confinement and substrate topography control cell migration in a 3D computational model, Commun. Phys. 2, 82 (2019).

[14] J. Park, D.-H. Kim, H.-N. Kim, C. J. Wang, M. K. Kwak, E. Hur, K.-Y. Suh, S. S. An, and A. Levchenko, Directed migration of cancer cells guided by the graded texture of the underlying matrix, Nat. Mater. 15, 792 (2016).

[15] D. Caballero, J. Comelles, M. Piel, R. Voituriez, and D. Riveline, Ratchetaxis: Long-range directed cell migration by local cues, Trends Cell Biol. 25, 815 (2015).

[16] S. E. Kim, M. S. Kim, Y. C. Shin, S. U. Eom, J. H. Lee, D.-M. Shin, S. W. Hong, B. Kim, J.-C. Park, B. S. Shin, D. Lim, and D.-W. Han, Cell migration according to shape of graphene oxide micropatterns, Micromachines 7, 186 (2016).

[17] E. L. Maout, S. L. Vecchio, A. Bhat, and D. Riveline, Directing cell migration on flat substrates and in confinement with microfabrication and microfluidics, in Microfluidics in Cell Biology Part B: Microfluidics in Single Cells, edited by M. Piel, D. Fletcher, and J. Doh, Vol. 147 of Methods in Cell Biology (Academic Press, New York, 2018), Chap. 7, pp. 109-132.
[18] D.-H. Kim, C.-H. Seo, K. Han, K. W. Kwon, A. Levchenko, and K.-Y. Suh, Guided cell migration on microtextured substrates with variable local density and anisotropy, Adv. Funct. Mater. 19, 1579 (2009).

[19] D.-H. Kim, P. P. Provenzano, C. L. Smith, and A. Levchenko, Matrix nanotopography as a regulator of cell function, J. Cell Biol. 197, 351 (2012).

[20] C. Heo, C. Jeong, H. S. Im, J. U. Kim, J. Woo, J. Y. Lee, B. Park, M. Suh, and T.-i. Kim, Cellular behavior controlled by bio-inspired and geometry-tunable nanohairs, Nanoscale $\mathbf{9}$, 17743 (2017).

[21] J. Park, D.-H. Kim, and A. Levchenko, Topotaxis: A new mechanism of directed cell migration in topographic ecm gradients, Biophys. J. 114, 1257 (2018).

[22] J. A. J. Wondergem, M. Mytiliniou, F. C. H. de Wit, T. G. A. Reuvers, D. Holcman, and D. Heinrich, Chemotaxis and topotaxis add vectorially for amoeboid cell migration, bioRxiv, https://doi.org/10.1101/735779.

[23] S. J. Ebbens and D. A. Gregory, Catalytic janus colloids: Controlling trajectories of chemical microswimmers, Acc. Chem. Res. 51, 1931 (2018).

[24] D. Selmeczi, S. Mosler, P. H. Hagedorn, N. B. Larsen, and H. Flyvbjerg, Cell motility as persistent random motion: Theories from experiments, Biophys. J. 89, 912 (2005).

[25] G. Volpe, I. Buttinoni, D. Vogt, H.-J. Kümmerer, and C. Bechinger, Microswimmers in patterned environments, Soft Matter 7, 8810 (2011).

[26] Y. Fily, A. Baskaran, and M. F. Hagan, Dynamics of selfpropelled particles under strong confinement, Soft Matter 10, 5609 (2014).

[27] Y. Fily, A. Baskaran, and M. F. Hagan, Dynamics and density distribution of strongly confined noninteracting nonaligning self-propelled particles in a nonconvex boundary, Phys. Rev. E 91, 012125 (2015).

[28] M. Khatami, K. Wolff, O. Pohl, M. R. Ejtehadi, and H. Stark, Active Brownian particles and run-and-tumble particles separate inside a maze, Sci. Rep. 6, 37670 (2016).

[29] P. Galajda, J. Keymer, P. Chaikin, and R. Austin, A wall of funnels concentrates swimming bacteria, J. Bacteriol. 189, 8704 (2007).

[30] L. Angelani and R. D. Leonardo, Geometrically biased random walks in bacteria-driven micro-shuttles, New J. Phys. 12, 113017 (2010).

[31] S. A. Mallory, C. Valeriani, and A. Cacciuto, Curvature-induced activation of a passive tracer in an active bath, Phys. Rev. E 90, 032309 (2014).

[32] L. Angelani, R. Di Leonardo, and G. Ruocco, Self-Starting Micromotors in a Bacterial Bath, Phys. Rev. Lett. 102, 048104 (2009).

[33] S. Pattanayak, R. Das, M. Kumar, and S. Mishra, Enhanced dynamics of active brownian particles in periodic obstacle arrays and corrugated channels, Eur. Phys. J. E 42, 62 (2019).

[34] C. Reichhardt and C. J. Olson Reichhardt, Active matter transport and jamming on disordered landscapes, Phys. Rev. E 90, 012701 (2014).

[35] M. Zeitz, K. Wolff, and H. Stark, Active Brownian particles moving in a random Lorentz gas, Eur. Phys. J. E 40, 23 (2017).

[36] A. Morin, D. Lopes Cardozo, V. Chikkadi, and D. Bartolo, Diffusion, subdiffusion, and localization of active colloids in random post lattices, Phys. Rev. E 96, 042611 (2017). 
[37] O. Chepizhko and T. Franosch, Ideal circle microswimmers in crowded media, Soft Matter 15, 452 (2019).

[38] G. Volpe and G. Volpe, The topography of the environment alters the optimal search strategy for active particles, Proc. Natl. Acad. Sci. 114, 11350 (2017).

[39] C. Bechinger, R. Di Leonardo, H. Löwen, C. Reichhardt, G. Volpe, and G. Volpe, Active particles in complex and crowded environments, Rev. Mod. Phys. 88, 045006 (2016).

[40] A. Zöttl and H. Stark, Emergent behavior in active colloids, J. Phys.: Condens. Matter 28, 253001 (2016).

[41] M. N. Popescu, W. E. Uspal, C. Bechinger, and P. Fischer, Chemotaxis of active Janus nanoparticles, Nano Lett. 18, 5345 (2018).

[42] S. Saha, S. Ramaswamy, and R. Golestanian, Pairing, waltzing and scattering of chemotactic active colloids, New J. Phys. 21, 063006 (2019).

[43] E. A. Novikova, M. Raab, D. E. Discher, and C. Storm, Persistence-Driven Durotaxis: Generic, Directed Motility in Rigidity Gradients, Phys. Rev. Lett. 118, 078103 (2017).

[44] C. Lozano and C. Bechinger, Diffusing wave paradox of phototactic particles in traveling light pulses, Nat. Commun. 10, 2495 (2019).

[45] M. S. Davies Wykes, X. Zhong, J. Tong, T. Adachi, Y. Liu, L. Ristroph, M. D. Ward, M. J. Shelley, and J. Zhang, Guiding microscale swimmers using teardrop-shaped posts, Soft Matter 13, 4681 (2017).

[46] J. Tong and M. Shelley, Directed migration of microscale swimmers by an array of shaped obstacles: Modeling and shape optimization, SIAM J. Appl. Math. 78, 2370 (2018).

[47] L. Angelani, A. Costanzo, and R. D. Leonardo, Active ratchets, Europhys. Lett. 96, 68002 (2011).

[48] B.-q. Ai, Q.-y. Chen, Y.-f. He, F.-g. Li, and W.-r. Zhong, Rectification and diffusion of self-propelled particles in a twodimensional corrugated channel, Phys. Rev. E 88, 062129 (2013).

[49] B.-Q. Ai and J.-C. Wu, Transport of active ellipsoidal particles in ratchet potentials, J. Chem. Phys. 140, 094103 (2014).

[50] P. K. Ghosh, V. R. Misko, F. Marchesoni, and F. Nori, SelfPropelled Janus Particles in a Ratchet: Numerical Simulations, Phys. Rev. Lett. 110, 268301 (2013).

[51] X. Ao, P. Ghosh, Y. Li, G. Schmid, P. Hänggi, and F. Marchesoni, Active Brownian motion in a narrow channel, Eur. Phys. J.: Spec. Top. 223, 3227 (2014).

[52] E. Yariv and O. Schnitzer, Ratcheting of Brownian swimmers in periodically corrugated channels: A reduced Fokker-Planck approach, Phys. Rev. E 90, 032115 (2014).

[53] J. Katuri, D. Caballero, R. Voituriez, J. Samitier, and S. Sanchez, Directed flow of micromotors through alignment interactions with micropatterned ratchets, ACS Nano 12, 7282 (2018).

[54] H. Takagi, M. J. Sato, T. Yanagida, and M. Ueda, Functional analysis of spontaneous cell movement under different physiological conditions, PLoS ONE 3, 1 (2008).

[55] L. Li, E. C. Cox, and H. Flyvbjerg, 'Dicty dynamics': Dictyostelium motility as persistent random motion, Phys. Biol. 8, 046006 (2011).

[56] P. Denissenko, V. Kantsler, D. J. Smith, and J. KirkmanBrown, Human spermatozoa migration in microchannels reveals boundary-following navigation, Proc. Natl. Acad. Sci. 109, 8007 (2012).
[57] V. Kantsler, J. Dunkel, M. Polin, and R. E. Goldstein, Ciliary contact interactions dominate surface scattering of swimming eukaryotes, Proc. Natl. Acad. Sci. 110, 1187 (2013).

[58] G. Yu, J. Feng, H. Man, and H. Levine, Phenomenological modeling of durotaxis, Phys. Rev. E 96, 010402(R) (2017).

[59] C. R. Doering, X. Mao, and L. M. Sander, Random walker models for durotaxis, Phys. Biol. 15, 066009 (2018).

[60] T. Chinnasamy, J. L. Kingsley, F. Inci, P. J. Turek, M. P. Rosen, B. Behr, E. Tüzel, and U. Demirci, Guidance and self-sorting of active swimmers: $3 \mathrm{~d}$ periodic arrays increase persistence length of human sperm selecting for the fittest, Adv. Sci. 5, 1700531 (2018).

[61] I. Niculescu, J. Textor, and R. J. de Boer, Crawling and gliding: A computational model for shape-driven cell migration, PLoS Comput. Biol. 11, 1 (2015).

[62] A. Pototsky and H. Stark, Active Brownian particles in twodimensional traps, Europhys. Lett. 98, 50004 (2012).

[63] M. Hennes, K. Wolff, and H. Stark, Self-Induced Polar Order of Active Brownian Particles in a Harmonic Trap, Phys. Rev. Lett. 112, 238104 (2014).

[64] A. P. Solon, M. E. Cates, and J. Tailleur, Active Brownian particles and run-and-tumble particles: A comparative study, Eur. Phys. J.: Spec. Top. 224, 1231 (2015).

[65] O. Dauchot and V. Démery, Dynamics of a Self-Propelled Particle in a Harmonic Trap, Phys. Rev. Lett. 122, 068002 (2019).

[66] H. C. Berg and D. A. Brown, Chemotaxis in escherichia coli analysed by three-dimensional tracking, Nature (London) 239, 500 (1972).

[67] I. Berdakin, Y. Jeyaram, V. V. Moshchalkov, L. Venken, S. Dierckx, S. J. Vanderleyden, A. V. Silhanek, C. A. Condat, and V. I. Marconi, Influence of swimming strategy on microorganism separation by asymmetric obstacles, Phys. Rev. E 87, 052702 (2013).

[68] M. Mijalkov and G. Volpe, Sorting of chiral microswimmers, Soft Matter 9, 6376 (2013).

[69] T. Bertrand, Y. Zhao, O. Bénichou, J. Tailleur, and R. Voituriez, Optimized Diffusion of Run-And-Tumble Particles in Crowded Environments, Phys. Rev. Lett. 120, 198103 (2018).

[70] O. Chepizhko and F. Peruani, Diffusion, Subdiffusion, and Trapping of Active Particles in Heterogeneous Media, Phys. Rev. Lett. 111, 160604 (2013).

[71] D. Takagi, J. Palacci, A. B. Braunschweig, M. J. Shelley, and J. Zhang, Hydrodynamic capture of microswimmers into spherebound orbits, Soft Matter 10, 1784 (2014).

[72] T. Jakuszeit, O. A. Croze, and S. Bell, Diffusion of active particles in a complex environment: Role of surface scattering, Phys. Rev. E 99, 012610 (2019).

[73] T. Bretschneider, H. G. Othmer, and C. J. Weijer, Progress and perspectives in signal transduction, actin dynamics, and movement at the cell and tissue level: Lessons from dictyostelium, Interface Focus 6, 20160047 (2016).

[74] E. Sahai, Illuminating the metastatic process, Nat. Rev. Cancer 7, 737 (2007).

[75] P. Friedl and S. Alexander, Cancer invasion and the microenvironment: Plasticity and reciprocity, Cell 147, 992 (2011). 
[76] P. Friedl, S. Borgmann, and E.-B. Bröcker, Amoeboid leukocyte crawling through extracellular matrix: Lessons from the dictyostelium paradigm of cell movement, J. Leukocyte Biol. 70, 491 (2001).
[77] A. F. M. Marée, V. A. Grieneisen, and L. Edelstein-Keshet, How cells integrate complex stimuli: The effect of feedback from phosphoinositides and cell shape on cell polarization and motility, PLoS Comput. Biol. 8, 1 (2012). 